\title{
"I Fear for My Safety, but Want to Show Bravery for Others": Violence and Discrimination Concerns Among Transgender and Gender-Nonconforming Individuals After the 2016 Presidential Election
}

\author{
Cindy B. Veldhuis, PhD, Laurie Drabble, PhD, MSW, MPH, Ellen D.B. Riggle, PhD, \\ Angie R. Wootton, MSW, and Tonda L. Hughes, PhD, RN, FAAN ${ }^{1,5}$
}

\begin{abstract}
In general, transgender individuals report experiencing high levels of discrimination and violence, as well as high levels of safety concerns and a need to be vigilant to the safety of their environs. The stress that arises from these high levels of violence and discrimination has sizable effects on mental and physical health. The results of the 2016 presidential election raised concerns that the rhetoric used during the campaign and the potential rollback of rights could result in higher rates of violence and discrimination against transgender and gender-nonconforming individuals. In the current study, we conducted an internet-based mixed methods study among a national convenience sample $(N=242)$ to better understand transgender, genderqueer, and gender-nonconforming individuals' concerns about, and experiences with, discrimination, violence, and hate crimes after the 2016 presidential election. Data for the current study were collected between December 2016 and May 2017. Quantitative analyses revealed that participants reported high levels of election-related concerns, including high levels of concerns about safety and discrimination since the election. Furthermore, a majority of respondents had been directly exposed to hate speech and violence. Qualitative responses centered around concerns related to the emboldening and legitimization of hate speech and violence, and the effects on respondents and their relationships. Our findings suggest a need for intervention and prevention efforts aimed at improving resiliency, as well as the need for continuing existing supportive policies, enacting policies on federal and state levels to document antitrans violence, and passing nondiscrimination legislation inclusive of gender identity.
\end{abstract}

Keywords: hate crimes, transgender individuals, safety, discrimination

\section{Introduction}

$\mathbf{T}$ RANSGENDER INDIVIDUALS REPORT experiencing high levels of violence, including harassment, sexual violence, and physical assault. Furthermore, many transgender individuals feel as though they must constantly evaluate their environments to determine whether they are safe (Levitt and Ippolito 2013). For example, in an early study of transgender people in Chicago, $56 \%$ reported feeling unsafe in public, and $43 \%$ reported feeling uncomfortable in public because of their gender identity (Kenagy and Bostwick 2005). Experiencing violence, discrimination, and feeling generally unsafe may raise fears of future discrimination and victimization leading to high levels of stress, including anticipatory stress (Reisner et al. 2015).

Minority stress theory suggests that sexual or gender minority status is associated with higher levels of stress due to marginalization and stigmatization (Meyer 1995, 2003). Gender minorities, particularly those whose gender nonconformity is visible, have been shown to be at increased risk of victimization and discrimination, thus increasing their experiences of stress (Hendricks and Testa 2012; Martin-Storey 2016; Testa et al. 2012). Individuals who are transgender or gender nonconforming also experience

\footnotetext{
${ }^{1}$ School of Nursing, Columbia University, New York, New York.

${ }^{2}$ School of Social Work, San José State University, San Jose, California.

${ }^{3}$ Political Science and Gender and Women's Studies, University of Kentucky, Lexington, Kentucky.

${ }^{4}$ Department of Medicine, University of California, San Francisco, California.

${ }^{5}$ College of Nursing, University of Illinois at Chicago, Chicago, Illinois.
} 
stress related to expectations of encountering rejection and discrimination (Hendricks and Testa 2012). Minority stress, including stigma-related stressors such as elevated expectations of violence, anticipated rejection, and experiencing discrimination (including daily, repeated, and acute incidents) increases the risk of psychological distress (e.g., depression and anxiety) and "health harming" coping behaviors, such as alcohol use, drug use, and suicide attempts (Bockting et al. 2013; Hatzenbuehler and Pachankis 2016; Hendricks and Testa 2012; Miller and Grollman 2015; Testa et al. 2012).

Stigma in the larger social and political environment puts transgender or gender-nonconforming individuals at increased risk of poor mental and physical health outcomes, thus creating health disparities. For example, higher levels of structural stigma (e.g., discriminatory policies, such as Defense of Marriage Act amendments, and nondiscrimination laws that do not include sexual orientation or gender identity) have been shown to be associated with higher rates of suicide attempts among transgender individuals (PerezBrumer et al. 2015). Although the Matthew Shepard and James Byrd, Jr. Hate Crimes Prevention Act (18 U.S.C. § 249 ) includes gender identity in federal law, only 17 states and the District of Columbia include gender identity in state hate crime laws. Twenty states and District of Columbia include gender identity in state nondiscrimination laws; however, current federal civil right laws do not explicitly include gender identity. Given the relatively few explicit legal rights available, transgender and gender-nonconforming individuals may feel particularly at risk related to recent changes in the political environment.

Experiencing discrimination has sizable effects on health and wellbeing, and inclusive laws do not eliminate discrimination. Even in states that include gender identity in at least some nondiscrimination laws, rates of perceived discrimination may remain high and have negative effects on wellbeing. For example, in a study of transgender individuals in Massachusetts - a state that includes gender identity in its statewide nondiscrimination law in all areas except, at the time of the study, public accommodations- $65 \%$ of those surveyed reported perceived discrimination in the past year. Those who reported discrimination also reported significantly higher levels of depressive symptoms and negative physical health symptomatology (Reisner et al. 2015).

Support for the LGBTQ (Lesbian, Gay, Bisexual, Transgender, Queer) community as a whole has been increasing over time. For example, in 1996 only $27 \%$ of those surveyed by Gallup (2017) supported same-sex marriage, but in May 2017 64\% supported marriage equality and 72\% supported same-sex relationships). Despite a steady rise in support of same-sex relationships, there has been a recent increase in anti-LGBTQ legislation introduced in multiple states, and reports of hate crimes, bullying, and discrimination remain high (Herek 2015; see also Levy and Levy 2016; Stone 2016 for a history of legislation). Transgender and gendernonconforming individuals have fewer legal rights than LGB individuals (Movement Advancement Project 2017), and the transgender community may be at disproportionate risk for structural stigma enacted through laws. For example, proposed legislation that seeks to limit the rights of transgender people has increased in recent years, including laws designed to bar access to (or even criminalize) use of public facilities consistent with gender identity, enable discrimination in healthcare, allow religiously motivated discrimination, impede access to identification documents with appropriate gender designation, and restrict youth from full participation in school activities (American Civil Liberties Union 2017). The public debate that accompanies such legislation may increase levels of minority stress and worsen mental and physical health outcomes (Frost and Fingerhut 2016).

The results of the 2016 presidential election have raised concerns that gains in the rights of sexual and gender minorities made under President Barack Obama's administration would be limited or rescinded by President Donald Trump's administration (Hirsch et al. 2017; Veldhuis et al. 2017). Moreover, negative rhetoric against immigrants, liberals, women, and Muslims used during the 2016 presidential campaign raised concerns that already marginalized communities might experience increased levels of discrimination and violence under the Trump presidency (Hirsch et al. 2017; Veldhuis et al. 2017). In the current study, we use a mixed methods approach to better understand transgender, genderqueer, and gender-nonconforming individuals' concerns about, and experiences with, discrimination, violence, and hate crimes after the 2016 presidential election. Using quantitative analyses, we document concerns about the impact of the election outcome and experiences with hate crimes. Thematic analyses were conducted with narrative responses to open-ended survey questions to explore themes to describe these concerns.

\section{Materials and Methods}

Beginning in December 2016, 909 sexual minority women and trans* individuals who were at least 18 years of age completed an online survey about their perceptions of marriage equality and the 2016 presidential election. The term "trans*" is a relatively recent and more inclusive term in which the asterisk represents a wildcard field, encapsulating all individuals who do not identify as the gender they were assigned at birth or who have a queer gender identity (e.g., genderqueer, trans woman, trans man, nonbinary, transgender, and gender fluid individuals). Data collection is ongoing; data for the present study are from December 2016 to May 2017. Recruitment strategies were designed to reach individuals who identified as lesbian, bisexual, queer, same-sex attracted or something other than exclusively heterosexual, as well as individuals who identify as trans*.

All recruitment took place online, and the study was advertised as a study of "Marriage Recognition and Recent Political Events." Advertisements were placed on social media sites (Facebook and Twitter), listservs (e.g., professional organizations and community organizations), and websites aimed at sexual minority women and trans* individuals (e.g., AfterEllen, Autostraddle, and Curve). The current sample included only those who identified as trans*, genderqueer, or nonbinary $(N=242)$, including $14.5 \%$ $(n=35)$ who identified as trans men, $19.8 \%(n=48)$ as trans women, and $65.7 \%(n=159)$ as genderqueer or nonbinary. All study procedures were reviewed and approved by the San José State University's Institutional Review Board.

\section{Measures}

Data were collected through an online survey using Qualtrics. The survey included both quantitative survey items 
and open-ended questions; all items were created for this study. Development of survey items were informed by prior research on minority stress (Hatzenbuehler 2009; Riggle et al. 2010); as such, they were designed to capture both concerns and experiences along a continuum from proximal (e.g., individual and interpersonal) and distal (e.g., community climate and media) levels.

Respondents completed items about the 2016 presidential election by selecting whether they had experienced any of five anti-LGBTQ events since the election: hate speech, discrimination, property crimes, violence, or threats or harassment. Response options were (yes/no) for four categories of experiences: experienced by participant personally; witnessed the event happening to someone else; heard about the event firsthand (e.g., someone I know or in my neighborhood; and heard from the news, social media, or other sources). Respondents were also asked about specific concerns related to the election by selecting "the category that best reflects your level of concern or feelings compared to how you felt before the election," using a 7-category scale (much higher, moderately higher, slightly higher, about the same, slightly lower, moderately lower, and much lower). Examples include: "concerns about LGBTQ rights," "concerns about my safety," and "concerns about the safety of others." Three items addressed individual reactions, including "feelings of sadness or depression," "feelings of anxiety," and "comfort with my identity."

Our question on gender identity is partially based on recommendations from The Williams Institute GenIUSS group (The GenIUSS Group 2013). We asked respondents to indicate their current gender identity by selecting "all that apply": female/woman; male/man; trans woman; trans man; gender nonconforming, genderqueer, nonbinary; and other (with the option to provide details or other ways in which participants identify). "Nonbinary" was added based on recommendations from participants in the study. Respondents were asked to indicate their sexual identity in response to a question based on the modified Kinsey scale and used in multiple studies (e.g., Hughes et al. 2015; only lesbian or gay; mostly lesbian or gay; bisexual; mostly heterosexual; only heterosexual; queer, questioning; or other, with the option to provide details or other ways in which participants identify). Demographics included age, relationship status, race/ethnicity, education, employment status, and gender of partner (for those in relationships).

\section{Analyses}

Qualitative analyses. Narrative data for this study were drawn primarily from an open-ended question inviting participants to "share with us your thoughts or concerns related to the current political and social environment." Participants were also asked, "In what ways has the election affected your relationships (e.g., intimate relationships, familial relationships, and relationships with friends)." Because some responses to this question overlapped with the research question for the current article (e.g., stigma-related concerns, perceptions, and expectations), these data were included in analyses.

Narrative responses were compiled for those who responded to at least one of the open-ended questions ( $n=124$ out of 242 responses). There were no significant demographic (i.e., age, race/ethnicity, gender identity, education, or employment) or emotional reaction (i.e., feelings of sadness or depression, or feelings of anxiety) differences between those who provided narrative responses compared with those who did not. Length of responses varied from one sentence to multiple paragraphs and ranged from 2 to 406 words.

Using Microsoft Word and Microsoft Excel, we conducted a thematic analysis of all narrative responses to identify patterned responses or meanings associated with the research question. Specifically, we used an experiential thematic analysis approach (Braun and Clarke 2013): first, responses were read and notes made to indicate when the response contained a meaning related to election-related concerns; second, responses were read for meaning and the meaning summarized; third, the authors agreed on general categories and definitions to capture the range of meanings; and, fourth, the individual meanings were categorized and grouped into larger themes. Minority stress theory was used broadly as a sensitizing concept in the development of a provisional coding guide, which included attention to the interplay of concerns on individual, interpersonal, community, and societal levels. Three of the authors used the guide to code a subsample of interviews, and then refined the coding guide to continue the iterative process of reviewing, defining, and naming categories of responses. Creswell and Poth (2018) suggest using at least two validation strategies in a qualitative study. The two primary validation strategies in the current study centered on engaging in reflexivity and conducting an audit of the research process: (1) the first four authors continued an open critical dialog about the emerging categories throughout the analysis, and (2) the qualitative findings were examined and verified by the last author (Creswell and Poth 2018).

Quantitative analyses. We had no a priori hypotheses for the quantitative analyses, thus these analyses are presented for descriptive purposes and as additional information to aid in the interpretation of our qualitative analyses. Univariate analyses were used to examine the distributions of election concerns by gender identity. We fit unadjusted ANOVA models to the data to examine differences in election concerns by gender identity. Because we had no a priori hypotheses, we ran all pairwise comparisons to examine gender identity differences. Because we had six pairwise comparisons for each of our election-related outcomes, we considered using a Bonferroni correction to reduce the risk of false positives; however, that would have reduced our critical $p$-value to 0.0083 , which may in turn have led to a risk of false negatives. Instead, because the quantitative results are intended to be descriptive and supplementary to our qualitative findings, when the pairwise comparisons are $<0.05$, we have reported the exact $p$-value, and interpreted them cautiously. For all analyses SPSS version 23 statistical software was used.

\section{Description of the sample}

See Table 1 for a description of the sample. Participants in our study came from 38 states in the United States, with the highest proportions coming from California $(21.9 \%)$, Illinois (7\%), Massachusetts (5.8\%), and New York (4.1\%). Most reported living in a large city $(45.8 \%)$ or in a medium- 
Table 1. Demographic Characteristics of the Sample

\begin{tabular}{|c|c|c|}
\hline & $\mathrm{n}$ & $\%$ \\
\hline \multicolumn{3}{|l|}{ Sexual identity } \\
\hline Lesbian/mostly lesbian & 91 & 37.8 \\
\hline Bisexual/mostly heterosexual & 60 & 24.9 \\
\hline Queer or other & 90 & 37.3 \\
\hline \multicolumn{3}{|l|}{ Gender identity } \\
\hline Trans woman & 48 & 19.8 \\
\hline Trans man & 35 & 14.5 \\
\hline Nonbinary/genderqueer & 159 & 65.7 \\
\hline \multicolumn{3}{|l|}{ Race/ethnicity } \\
\hline White & 171 & 85.1 \\
\hline African American & 3 & 1.2 \\
\hline Latinx & 8 & 3.3 \\
\hline American Indian/Alaska native & 3 & 1.2 \\
\hline Asian/Pacific Islander & 15 & 6.2 \\
\hline Other & 1 & 0.4 \\
\hline \multicolumn{3}{|l|}{ Education } \\
\hline$<$ High school & 3 & 1.5 \\
\hline High school/GED & 8 & 3.3 \\
\hline Technical/vocational schooling & 2 & 0.8 \\
\hline Some college/currently attending & 46 & 22.9 \\
\hline 2-year college degree & 3 & 1.5 \\
\hline 4-year college degree & 60 & 29.9 \\
\hline Currently enrolled in graduate school & 16 & 8.0 \\
\hline Graduate or professional degree & 63 & 31.3 \\
\hline \multicolumn{3}{|l|}{ Employment } \\
\hline Full time & 49 & 54.4 \\
\hline Part time & 21 & 23.3 \\
\hline Unemployed & 5 & 5.6 \\
\hline In school & 4 & 4.4 \\
\hline Retired & 1 & 0.4 \\
\hline Other & 10 & 11.1 \\
\hline \multicolumn{3}{|l|}{ Gender identity of partner } \\
\hline Female & 68 & 66.0 \\
\hline Male & 13 & 12.6 \\
\hline Trans & 12 & 11.7 \\
\hline Nonbinary & 10 & 9.7 \\
\hline \multicolumn{3}{|l|}{ Relationship status } \\
\hline Single or dating & 85 & 35.1 \\
\hline In a committed relationship (no legal status) & 87 & 36.0 \\
\hline Legal status other than marriage & 2 & 0.8 \\
\hline Married & 44 & 18.2 \\
\hline Separated/divorced & 5 & 2.1 \\
\hline Other & 19 & 17.9 \\
\hline \multicolumn{3}{|l|}{ Cohabiting } \\
\hline Yes & 102 & 68.9 \\
\hline No & 46 & 31.1 \\
\hline \multicolumn{3}{|l|}{ Current residence } \\
\hline Large city & 92 & 45.8 \\
\hline Suburb of large city & 29 & 14.4 \\
\hline Medium-sized city $(50,000-250,000)$ & 44 & 21.9 \\
\hline Small city or town (under 50,000 ) & 30 & 14.9 \\
\hline Rural & 4 & 2.0 \\
\hline Other & 2 & 1.0 \\
\hline
\end{tabular}

sized city $(21.9 \%)$. More than half $(54.5 \%)$ reported that their city/town had an LGBT nondiscrimination policy, and a large proportion reported that their place of employment $(79.5 \%)$ or their school $(77.8 \%)$ had nondiscrimination policies. The mean age of respondents in the sample was
29.9 (standard deviation $[\mathrm{SD}]=9.0$; range $18=65$ ). $\mathrm{Re}-$ spondents self-identified as white $(85.1 \%)$, Asian $(7.5 \%)$, Latinx (gender-neutral term for Latino/Latina; $4.0 \%$ ), black $(1.5 \%)$, American Indian/Alaska Native (1.5\%), and other $(0.5 \%)$. Approximately one-third had a graduate degree $(31.3 \%)$, and one-third had a college degree (29.9\%). Most respondents reported working full time $(54.4 \%)$. Only $6.5 \%$ reported having children under the age of 18 living in the home, but $18.9 \%$ were planning to have children.

\section{Results}

Quantitative findings document high levels of exposure to hate speech, discrimination, and violence, and increased levels of concern about health and safety at individual, interpersonal, and community/societal levels. Qualitative findings, described after the summary of quantitative findings, were consistent with quantitative findings, and provide insight about the specific violence-related concerns, perceptions, and expectations of respondents associated with the election.

\section{Quantitative findings}

Prevalence of exposure to hate speech, discrimination, and violence. Table 2 summarizes frequencies of exposure to hate speech, discrimination, and violence against LGBTQ people since the 2016 presidential election. Overall, a large proportion of respondents $(81.4 \%)$ reported hearing about hate crimes against LGBTQ people in the news or on social media since the election, ranging from a high of $82.9 \%$ who reported hearing about violence against LGBTQ people to a low of $77.7 \%$ who had heard about property crimes. Across hate crime types, more than half $(55.8 \%)$ reported hearing firsthand about any type of hate crimes, $37.2 \%$ reported having witnessed any type of hate crimes, and $29.8 \%$ reported experiencing any type of hate crimes personally since the election. We found few group differences. Half of trans men $(50 \%)$ reported having experienced hate speech compared with $24.4 \%$ of trans women and $24.4 \%$ of genderqueer/ nonbinary individuals, $\chi^{2}(2, N=198),=6.445, p<0.05$, and $35 \%$ of trans men reported having experienced discrimination since the election compared with $17.8 \%$ of trans women and $12.7 \%$ of genderqueer/nonbinary individuals, $\chi^{2}(2$, $N=191),=6.418, p<0.05$. No other group differences were found.

Table 2. Exposure to Hate Speech, Discrimination, and Violence Since the 2016 Presidential Election

\begin{tabular}{|c|c|c|c|c|c|c|c|c|}
\hline & \multicolumn{2}{|c|}{$\begin{array}{c}\text { News/social } \\
\text { media }\end{array}$} & \multicolumn{2}{|c|}{$\begin{array}{l}\text { Heard } \\
\text { firsthand }\end{array}$} & \multicolumn{2}{|c|}{ Witnessed } & \multicolumn{2}{|c|}{ Experienced } \\
\hline & $\mathrm{n}$ & $\%$ & $\mathrm{n}$ & $\%$ & $\mathrm{n}$ & $\%$ & $\mathrm{n}$ & $\%$ \\
\hline Hate speech & 178 & 80.9 & 109 & 51.7 & 69 & 34.5 & 54 & 27.3 \\
\hline Discrimination & 175 & 79.2 & 90 & 43.5 & 48 & 24.0 & 31 & 16.2 \\
\hline Property crimes & 171 & 77.7 & 55 & 27.1 & 17 & 9.0 & 4 & 2.2 \\
\hline Violence & 184 & 82.9 & 53 & 26.1 & 11 & 5.9 & 6 & 3.3 \\
\hline Threats/harassment & 175 & 79.9 & 95 & 45.7 & 42 & 21.1 & 39 & 19.8 \\
\hline All types (total) & 197 & 81.4 & 135 & 55.8 & 90 & 37.2 & 72 & 29.8 \\
\hline
\end{tabular}


Increases in election-related concerns. Tables 3 and 4 display mean and SDs for election-related concerns. Overall, the proportion of respondents who reported higher ("moderately" and "much higher" combined) concerns since the results of the election was large: $78.5 \%$ reported high levels of concerns about LGBT rights, $66.1 \%$ reported concerns about their own safety, and $82.2 \%$ reported high levels of concerns about others' safety. More than $75 \%$ of the sample reported a high level of concern about discrimination $(77.7 \%)$, and more than half reported being concerned about social acceptance $(52.1 \%)$. Slightly less than half were concerned about social rejection $(49.5 \%)$ and $13.2 \%$ were concerned about the effects of the election on their own identity. More than half of the sample reported high levels of sadness or depression $(62.0 \%)$ and anxiety (64.0\%) since the election. Smaller proportions reported concerns about their relationships with their families $(24.4 \%)$ and with their partner/spouse $(8.7 \%)$.

There were few group differences among the electionrelated concerns. There was a significant effect of gender identity on concerns about the safety of others, $F$ (2, $203)=3.752, p<0.05$. Trans women $(M=6.88, \mathrm{SD}=0.33$; $p=0.026)$ and genderqueer/nonbinary individuals $(M=6.88$, $\mathrm{SD}=0.37 ; p=0.023)$ were significantly more concerned about the safety of others than were trans men $(M=6.59$, $\mathrm{SD}=1.12)$. A different pattern emerged for concerns for oneself, $F(2,203)=3.667, p<0.05$; trans women $(M=6.59$, $\mathrm{SD}=0.63$ ) were significantly more concerned about their own safety than were trans men $(M=6.00, \mathrm{SD}=1.19$; $p<0.014)$ and genderqueer/nonbinary individuals $(M=6.19$, $\mathrm{SD}=0.92 ; p=0.020)$. There were no other group differences in election-related concerns.

\section{Qualitative descriptions of concerns}

Six themes emerged from the analysis: (1) concerns about increased bias and discrimination against transgender and LGB people; (2) anticipated increase in violence against transgender and gender-nonconforming people, including threats to personal safety; (3) concerns that rollbacks of rights and supportive policies would threaten transgender individuals' wellbeing; (4) experienced need to reassess boundaries and safety in relationships with others; (5) concerns about visibility of a marginalized identity; and, (6) perceived negative impact on psychological wellbeing.

Concerns about increased bias and discrimination against transgender and LGB people. Respondents worried about increases in hate in the broader social environment, or as one transgender woman expressed, "I worry about what feels like a reactionary shift in our culture that is already inciting hatred and violence, not just toward LGBT people but toward all women, people of color, people of non-Christian faith, and other marginalized groups." Respondents felt that the election had created a risky environment:

The night of the election was the first time I've felt frightened by the nation around me. I've been very privileged to experience relatively low levels of discrimination or harassment due to my identity, and I believed that the world was becoming more tolerant and accepting of people with different perspectives and experiences. The election of an individual who promotes racist, ableist, and misogynistic ideas made me reevaluate my view of the world becoming safer and more accepting. (genderqueer respondent).

Whereas some respondents felt that LGBTQ people would be targeted (literally, "I'm so scared that we will be tracked, that we will have crosshairs on our backs...."; genderqueer respondent); others felt that the Trump administration would legitimize hateful ideologies and actions: "It is one thing to have laws that discriminate against you, and another that has powerful people modeling actions and words that devalue you as a person and encourage others to do so," stated a genderqueer woman. The election left many feeling that it is not safe "for people like me in this country anymore" (trans woman). Some expressed concerns about interacting with others: "It makes me feel like I cannot trust anyone around me or believe that their beliefs about who I am are anything but awful" (genderqueer respondent).

Impact of expected violence and threats to personal safety. Respondents described an increase in feeling personally unsafe or uncomfortable in their local community since the election. For example, one trans woman noted, "Hate groups and hating individuals are now more empowered to act outside the law, so the likelihood of assault feels much higher." Similarly, a gender-nonconforming respondent described feeling "very concerned about my personal welfare based on the type of behavior and discrimination that has been brought to the forefront and encouraged for the average citizen" and, specifically, "very concerned about my safety and my personal rights in the coming administration." Respondents frequently shared specific examples of actual hate speech or hate crimes. For example, one trans woman observed,

Since the election, I've had friends be told that gay lives don't matter anymore. I know people who have

Table 3. Concerns About Rights, Discrimination, Safety, and Social Acceptance/Rejection Since the 2016 Election, Scale from 1 (Much Lower to 7 (Much Higher)

\begin{tabular}{|c|c|c|c|c|c|c|c|c|c|c|c|c|}
\hline & \multicolumn{2}{|c|}{$L G B T$ rights } & \multicolumn{2}{|c|}{ My safety } & \multicolumn{2}{|c|}{ Discrimination } & \multicolumn{2}{|c|}{ Social acceptance } & \multicolumn{2}{|c|}{ Social rejection } & \multicolumn{2}{|c|}{ My identity } \\
\hline & M & $S D$ & M & $S D$ & M & $S D$ & M & $S D$ & M & $S D$ & M & $S D$ \\
\hline Trans women & 6.71 & 0.75 & 6.59 & 0.63 & 6.68 & 0.57 & 5.98 & 1.01 & 4.54 & 1.00 & 4.22 & 1.31 \\
\hline Trans men & 6.55 & 1.15 & 6.00 & 1.49 & 6.52 & 1.12 & 5.34 & 1.42 & 5.00 & 1.54 & 3.86 & 1.30 \\
\hline Genderqueer/nonbinary & 6.68 & 0.67 & 6.19 & 0.92 & 6.61 & 0.66 & 5.79 & 1.08 & 4.72 & 1.33 & 4.23 & 1.23 \\
\hline Total & 6.67 & 0.77 & 6.24 & 0.99 & 6.61 & 0.72 & 5.76 & 1.13 & 4.73 & 1.30 & 4.18 & 1.26 \\
\hline
\end{tabular}

LGBT, lesbian, gay, bisexual, transgender; SD, standard deviation. 
Table 4. Relationship and Psychological Concerns Since the 2016 Election, SCAle From 1 (Much Lower) to 7 (Much Higher)

\begin{tabular}{|c|c|c|c|c|c|c|c|c|}
\hline & \multicolumn{2}{|c|}{ Family relationship } & \multicolumn{2}{|c|}{ Partner relationship } & \multicolumn{2}{|c|}{ Sadness/depression } & \multicolumn{2}{|c|}{ Anxiety } \\
\hline & M & $S D$ & M & $S D$ & M & $S D$ & M & $S D$ \\
\hline Trans women & 4.54 & 1.00 & 4.22 & 0.94 & 6.02 & 1.23 & 6.24 & 0.94 \\
\hline Trans men & 5.00 & 1.54 & 4.21 & 1.26 & 5.72 & 1.75 & 5.90 & 1.47 \\
\hline Genderqueer/nonbinary & 4.72 & 1.33 & 4.18 & 1.02 & 6.11 & 0.92 & 6.28 & 0.94 \\
\hline Total & 4.73 & 1.30 & 4.19 & 1.04 & 6.04 & 1.14 & 6.22 & 1.03 \\
\hline
\end{tabular}

been assaulted for being LGBTQ. I pass off as a straight, cisgender woman in public, but others aren't so lucky, and have faced harassment.

A respondent who identified as genderqueer also noted,

I'm from a red state originally (Idaho), and my friends there have reported harassment. One person I know there, a gay man, had his car vandalized with hate speech. My best friend was sexually harassed on the street by men she didn't know immediately after the election. Someone I went to high school with, who's Latino, had a car try to hit him as he crossed the street and a man inside it yell, "We're coming for you!"

Many respondents described how the expectation of violence impacted their behavior or elevated their vigilance about safety. For example, one person who identified as gender nonconforming stated, "I live in a very rural and conservative area; the day after Election Night, I felt so hypervigilant and fearful even leaving my house." Another respondent who identified as gender nonconforming said, "I am scared shitless. My emotions run high, I am constantly on edge, and I'm very aware of my surroundings and the people around me." A participant who identified as gender nonconforming and who lived in a rural area noted that it was hard to trust anyone,

It makes me fearful for what will happen to me. It makes me feel like I cannot trust anyone around me or believe that their beliefs about who I am are anything but awful. I know that this is irrational, but there is just such a strong feeling in rural conservative areas that lets someone like me know that "you don't belong" and in the absence of a community or people there who are vocal about opposing this sort of belief system, well-it just makes it a very very difficult place to live.

Another respondent, explained, "My romantic relationship with one of my partners who is also nonbinary makes me feel scared sometimes that we will get harassed when we're out together'" (nonbinary respondent).

Some talked about the effects of the election on their comfort with simply being out with their partner in public,

I think I was always afraid of holding hands in public, but now I definitely won't hold hands in public. I feel like my partner is saddened by this, but agrees. I think this pretty much sums up the political climate at the moment, it's hard to be authentic or open in an unfamiliar environment (genderqueer respondent).

Other respondents described feeling relatively safe in their local community, but described a substantial reduction in their assessment of safety outside of local boundaries, which is illustrated by one respondent, who explained, "The majority of the USA I fear to travel through; I fear being arrested or assaulted every day at a slightly higher level than before the election, and I was extremely paranoid before then" (gender-nonconforming respondent). This theme was echoed in the observations of another trans woman, who stated, "I feel relatively safe and accepted in my community, but less safe or accepted than ever in my state as a whole." One participant noted that the concerns went far beyond violence: "My queerness impacts my daily life in subtler ways than overt violence-more in terms of what I have access to, what people assume of me, the institutional barriers we still have to face" (gender-nonconforming respondent).

Concerns that rollbacks of rights and supportive policies would threaten transgender individuals' health and safety. Respondents cited numerous concerns about policies that might change under the Trump administration. These policies included ones that specifically establish rights for people who are transgender (e.g., nondiscrimination laws) as well as more general policy areas (e.g., the Affordable Care Act [ACA]). Respondents were "worried," "scared," and "anxious" about how policy changes could negatively impact them, their family, and others. Respondents had significant concerns about the rollback of rights in local nondiscrimination policies, especially in the absence of a federal law. Access to public accommodations, including use of appropriate restroom facilities was also a concern for those who had transitioned. One respondent referred to several areas, "I personally worry about losing what few legal protections and status I hold as a transgender woman. I fear losing access to public accommodations (e.g., bathrooms), worry about what will happen to my healthcare and health insurance (and the nondiscrimination protections surrounding gender identity), and whether I'll be harassed out in public" (trans woman). A few respondents also worried about the passing of laws that would actively discriminate against transgender and LGBTQ people, for example, "I'm concerned about discriminatory laws and executive orders, and concerned about antidiscrimination protections being rolled back or halted" (genderqueer respondent).

For individuals who were in the process of, or had, transitioned, laws impacting access to medical care and gender identity on government documents were a concern. One genderqueer respondent wrote about these concerns, "I am afraid that I will not be able to access the mental and physical healthcare that I need to stay healthy. I am in the early stages of a gender transition. I had expected to be able to ease into the changes, especially socially and legally, and 
now I need to decide within the next week whether I want to change my passport now or potentially have an $\mathrm{F}$ on my passport for the next eight years." Another respondent, a trans man, had changed his gender identification on his passport immediately after the election out of fear of not being able to do so in the future.

Respondents also worried about more general policy impacts. For example, some worried about the rollback of marriage equality, "I'm quite worried that the social environment with regard to LGBT issues will deteriorate and that marriage equality will be, if not repealed, weakened through state laws" (genderqueer respondent). Others expressed concerns about access to healthcare in general, "My partner has a chronic illness, and we are both trans-fear about losing our medical coverage and medical access has cast a long shadow. We are afraid to lose our ACA health insurance, as we both have preexisting conditions (mine disqualified me from buying insurance for myself pre-ACA). It is hard to plan for a future knowing life-sustaining medical care may be ripped away" (trans man). There were also worries that, "the incoming U.S. administration will crash the economy, make it more difficult for the most disadvantaged to get what they need..." (trans woman). Respondents also expressed concerns about the civil rights of all people, "It seems like all of the leadership in the incoming administration is strongly against the civil rights of all diverse people. I fear that these people will work to destroy everything that we have spent the last fifty years gaining" (trans woman).

Assessing threat and safety in relationships with others. Many respondents described increased relationship strain as a result of the election. As one gendernonconforming respondent described, "This election has made all of my close relationships, especially with my partner, more stressful because we are all very concerned about what it will mean for ourselves, people we care about, the country, and the world."

Respondents frequently described postelection rifts with family or community. For example, one queer trans man explained the emotional toll of communicating with his family postelection, stating, "All my family voted for Trump; I have to explain to them nearly daily, in every interaction, why this is painful and why I'm scared of the new administration." Other respondents articulated deep declines in trust of others, typified by one gender-nonconforming respondent, who commented, "I feel like I can no longer trust some members of my community and even my family," and a trans man, who reassessed trust of some family members, noting, "whether or not they know about my issues, they have made it clear that they would not support me by voting for someone who discriminates against my kind." Many respondents described feeling increased rejection or distance from family, such as one trans man who commented, "Having been estranged from my immediate and extended family long before the 2016 election, the circumstances for my ostracism have been heightened to a new extent of transphobia, homophobia, and racism." Similarly, a gender-nonconforming respondent described feeling disconnected from family.

It is easy to feel like I'm out here on my own when many members of my family lean to the right_-and even those who don't are very uninformed and live in a "bubble" that renders them unable to sincerely care about people who are "different" from themselves.

Many respondents described actively severing connections with family or other individuals in their social networks. For example, one gender-nonconforming respondent stated, "If I find out someone is a Republican I basically have to write them off as being dedicated to imaginary ideals that completely erase, ignore, stigmatize, and oppressively punish me and the ways [I'm] forced to interact with all bureaucracies and institutions." Similarly, another gender-nonconforming respondent declared, "I have had less contact with my father due to his voting for Trump. It makes me so sad and angry that he could do such a thing having a queer kid, and it feels like he did it out of spite-i.e., out of an anxiety that, as someone who started out working class, what he has is somehow being encroached upon by 'others' of various kinds." A trans man, one of the only respondents who favored the election outcome, commented, "If my friends knew I voted for Trump, I'd lose many, if not most, of them. My wife's family would be furious."

At the same time, respondents frequently described concurrent efforts among family and community members to address safety concerns and provide support. For example, one gender-nonconforming respondent aptly summarized this theme,

I feel closer to my friends. I feel closer to my mom as we try to support each other. Everyone is offering to drive people home or walk with people if they feel unsafe. Having to do so isn't what anyone wants, but we are so willing to protect each other.

Similarly, a trans man notes, "There has been such focused anger and resistance in my community and among my friends and family after the election." At the same time, this respondent observed "increasing calls for mutual aid and resource sharing" as well as "a real sense of support and solidarity." He concludes, "I feel scared, but I don't feel alone." Another nonbinary respondent perceived that everyone is trying hard to be extra caring for everybody else and noted that although "bio family is a mixed bag, I have a few relatives who are supportive of my gender identity, and they've expressed compassionate concern for my safety.",

Concerns about visibility and having multiple marginalized identities. Respondents worried about the effects of the election on their sexual and gender identity and their ability to be out, such as a trans man who stated, "I am afraid to tell others about my gender identity and my sexuality because of this election and the environment it has created. The election of our current president has proven that our world is not accepting of people like me and my significant other." Some felt as though coming out to others now was too dangerous, "I am frustrated that my hateful relatives feel so validated by this past election. Even before the primaries were over, they began using hate speech. I can't come out to them. If it was dangerous before, it's probably deadly, now" (genderqueer respondent). Respondents also raised concerns about "passing" as heterosexual or cis-gendered, "I worry for the safety of queers that don't pass at hetero" (genderqueer woman). Those who felt like they "pass" expressed some safety in that, "though I am a femme presenting queer 
person, I look to the outside world like a white woman, so I am significantly less scared for my own safety" (genderqueer respondent). Yet, others worried about the potential deleterious effects of visibility, "I feel like I'm very visibly queer/trans/gender-nonconforming and my anxiety levels about being out and about have just been SO high." (genderqueer respondent). One genderqueer respondent connected the freedom to be out and visible with protections afforded to the LGBTQ community by supportive policies, "I felt comfortable coming out only when I knew I had legal protection. If those laws are rolled back, I won't be able to respond to homophobia with anything other than my own personal convictions" (genderqueer).

At the same time, some saw their sexual and gender identity as a form of rebellion or resistance and expressed a desire to be visible as a statement, "I still would never want to take back my choice to live as myself, openly and proudly" (trans woman). Some wanted to be out to increase visibility of the community, to help others, and to get support, such as a trans woman who stated,

I have, in the last 3 months, come out to everyone and found familial support. I have also helped provide them with an anchor person. By being out, it means that if popular opinion turns further against me and those like me, they have someone to point out and say "that is my daughter/sister/niece, and she deserves the same rights as everyone else" (trans woman).

For some, the feelings of wanting to be visible clashed with fears for safety, creating some internal tension, "The election has both emboldened and diminished my desire to present as gender nonconforming. On one hand, I fear for my safety, but on the other, I would like to show bravery for others" (genderqueer respondent).

Respondents raised specific concerns about the effects of the election on those with multiple marginalized identities, "Survival has never been easy for people in the most marginalized communities within the umbrella of gender and sexual diversities, and I see that it's just getting harder at the moment" (genderqueer respondent). Respondents were particularly concerned about potential policies that might disproportionately affect LGBTQ people of color, "I'm fearful for the safety of my queer and brown siblings. The current political environment has empowered many folks who want to enact policies that directly threaten my livelihood, and it's hard to believe that it's going to be okay" (genderqueer respondent). Specific concerns were also raised for the safety of transgender people of color, "I am highly concerned for our Trans brothers and sisters, especially Trans people of color. Every day I wake up, it seems like another Trans POC's life has been taken away. 8 trans women have already been killed in 2017" (trans man).

Concerns about election-related impact on psychological wellbeing. Multiple respondents reported feeling fearful, depressed, and overwhelmed since the election, such as a trans woman who stated, "For me personally, all this pervasive fear and concern manifests as anxiety, depression, and nearly constant agitation" (trans woman). Some reported a shifting emotional landscape, "I seem to swing wildly between terror, hopelessness, and determination" (genderqueer respondent), whereas others reported over- whelming feelings of hopelessness, numbness, and immobilization. One gender-nonconforming respondent felt as though the election compounded already sizable psychological and health concerns, "I already have PTSD, oral health problems, and other healthcare problems due to being discriminated against, trafficked, assaulted for years, and tracked by white supremacy groups while I was economically disadvantaged and stuck in 'the south'."

Some reported thoughts of suicide, or feeling that they would be better off dead, "And I thought, Mike Pence believes that the only good tranny is a dead tranny, and he thinks the world would be better off if I were dead. He doesn't even believe I exist... I should just kill myself now" (trans woman). For one trans woman, the results of the election brought fears of a foreshortened future, "I'm very scared. I accept that, as a trans woman, I'm probably not going to live to see my $32^{\text {nd }}$ birthday because I will have been murdered, and I'm weirdly at peace with that."

\section{Discussion}

Overall, findings from this study document a high level of concerns about the effects of the election on the LGBTQ community broadly, and on transgender and gendernonconforming individuals specifically. Our findings suggest that fears of discrimination and violence are particularly high, which has negative implications for respondents' sense of safety, as well as their emotional wellbeing and physical health. In addition to safety-related fears, a large proportion of our sample has been exposed through news and social media outlets to reports of hate speech, discrimination, and violence targeting transgender people. Moreover, many respondents reported having witnessed or experienced discriminatory or violent events themselves since the election, suggesting that fear of discrimination and violence are being realized at least by some.

Narratives of study respondents suggest that the stigmatizing rhetoric of the presidential campaign coupled with the emboldening and legitimization of hate speech and violence likely activates minority stress, including the anticipation of future discrimination and violence. Among sexual and gender minorities, concerns about physical safety and rights exacerbates stress, thereby creating additional risk for health disparities. The effects of minority stress may be particularly keen in light of almost a decade of progress in attitudes toward, and protections and rights for, sexual and gender minorities. Furthermore, there is some evidence that minority stress may disproportionately affect transgender individuals, putting them at potentially higher risk compared with sexual minorities (Testa et al. 2015). More research is needed to understand the effects of increased stigma and structural stigma after a period of progress and improvements in societal attitudes (e.g., changes in the Department of Education policies from being supportive of gender identity appropriate facility use under the Obama administration to nonsupportive under the Trump administration). More research is also needed to understand the effects of legislative and policy changes by the Trump administration on the transgender community and LGBTQ community more broadly. For example, the exclusion of transgender individuals from serving in the military likely has negative effects on those in the military, as well as 
gender and sexual minorities more broadly through its stigmatizing message.

Transgender respondents in the current study described amplified attention to, and concerns about safety. Among those in our sample, trans men were most likely to report having experienced hate speech and discrimination, but trans women and genderqueer/nonbinary individuals were most likely to be concerned about the safety of themselves and others. These findings demonstrate a different pattern than those described in Stotzer's (2009) review of research on violence against transgender individuals. In general, across studies trans women reported higher rates of sexual violence (ranging from $23 \%$ to $69 \%$ ) than did trans men (30 to $35 \%$ ), and higher rates of violence in the home; trans women's reports of violence ranged from 65 to $67 \%$ and trans men's reports of violence ranged from 29 to 39\%. Yet, trans men were more likely than trans women to report feeling unsafe (85\% compared with $43 \%$; Kenagy and Bostwick 2005; Stotzer 2009). That our findings are not consistent with prior research may be due to differences in sampling techniques, thus more research is needed.

It was notable that many respondents described concerns about increased risk in the current social and political environment - both their own and others - in relation to the degree to which they "pass" as cisgender. Among transgender minorities who do not "pass" or who feel more visible, there is some evidence to suggest risks for violence and discrimination are higher (Martin-Storey 2016). Recent research suggests that for transgender adults, visually conforming to the gender binary may reduce risks for discrimination based on gender identity, and that among transgender individuals who are visually nonconforming, these high levels of discrimination may be associated with higher risk of posttraumatic stress disorder (PTSD) symptomatology (Reisner et al. 2016) and higher risk for suicide attempts (Miller and Grollman 2015).

In our study, transgender respondents expressed concerns about specific shifts in policy that they linked to their immediate and long-term health and wellbeing. Specifically, many respondents in our study feared that policy changes under the Trump administration would make it difficult or impossible for transgender individuals to align the gender markers on their identification documents with their gender identity, which could affect travel, employment, housing, and other rights and liberties (Levitt and Ippolito 2013). Given that transgender individuals are already at high risk of homelessness, workplace harassment, and discrimination, and overall have lower income despite higher levels of education, policies that further restrict rights may have disastrous effects on health and wellbeing of transgender individuals (Levitt and Ippolito 2013). Respondents additionally worried that the repeal of the Affordable Care Act would make gender reassignment surgery unobtainable. For many, and not only those who wish to "pass," concerns about safety are much higher and overlap with policy concerns. Future studies should follow changes in legislation and their impact on transgender individuals.

Among respondents in our study, concerns about relationships with family and with partners/spouses were moderate. In their qualitative responses, respondents discussed the rifts they felt with their family members who voted for Trump, or who simply failed to understand how his becoming president might have negative implications for them and for sexual and gender minorities as a whole. Respondents reported feeling unsafe, losing trust, and feeling a need to create boundaries (physical or emotional distance) with some family members. Given that in cases of violence against transgender individuals, the perpetrator is typically known to the victim (Stotzer 2009), more research is needed to determine whether familial risks and violence have increased since the election.

Transgender individuals with multiple marginalized identities are at high risk of both discrimination based on their multiple identities and higher risk for PTSD symptoms (Reisner et al. 2016). Among transgender individuals who have been murdered, $91 \%$ were people of color and most were low income (Stotzer 2009). Intersectionality thus plays a large role in violence against transgender individuals. Consistent with these findings, respondents in our studies worried both for themselves and for others with multiple marginalized identities, and the ways in which policy decisions by the new Presidential administration might disproportionately affect them, and lead to negative health outcomes-and even higher risk for mortality.

Notably, some respondents in our study saw their identities as gender minorities as a form of resistance. Although gender nonconformity broadly puts individuals at risk of stigma and discrimination, multiple people in our study refused to hide their identity or gender expression, despite their fears and concerns. Indeed, some discussed wanting to amplify their sexual identity and gender nonconformity and to be more "out" to increase visibility, despite the potential risks. Previous research on resiliency among transgender individuals suggests that pride in one's gender identity is a key factor in coping with minority stressors and overcoming traumatic events (Singh and McKleroy 2011). Being more "out" could also support increased connections to the transgender community specifically, as well as to the broader LGBTQ community, which may also foster resiliency and provide buffers against the impact of stressors (Singh and McKleroy 2011; Singh et al. 2011). More research is needed to examine the role of gender expression and resilience in this context.

The high levels of fear, sadness, and anxiety among respondents in our study, the feeling that their lives were unimportant to the new presidential administration (and a few mentions of suicidal thoughts or wondering if they would be better off dead) suggest a critical need for intervention and prevention efforts to address mental health risks and support resilience. Such efforts can equip transgender and gender-nonconforming individuals and communities with tools to help buffer the effects of the election to reduce risks of suicide attempts, lower risk of PTSD, and increase resilience and improve mental health functioning. For optimal outcomes, intervention and prevention efforts should aim to increase the resilience of not just individuals, but of communities as a whole (Meyer 2015).

\section{Limitations}

This study is among the first to examine the effects of the U.S. 2016 presidential election on transgender, genderqueer, and gender-nonconforming individuals, and more broadly, the perceived effects of social and political climate on the risks of discrimination and violence. Nevertheless, there are 
several limitations that limit the generalizability of our findings. First, although we asked respondents to choose as many gender identities as applied, we did not ask about their sex assigned at birth. Thus, some transgender individuals may have indicated they were male or female, without also indicating they were transgender, genderqueer, or gender nonconforming, in which case their data were excluded from these analyses. Furthermore, we did not ask questions about how participants in our study presented nor how they were perceived vis-à-vis their gender presentation and identity. Given that people who are more nonconforming may be at higher risk of victimization, this is an important direction for future research in this area. Second, the study relied on a convenience sample which limits generalizability of the findings to the broader transgender population. Third, despite attempts to increase sample diversity, the overwhelming majority of respondents identified as white and reported higher levels of education than the national average. Fourth, the survey did not include measures related to health or validated measures of psychological distress, so it is not possible to assess the impact of increased concerns on health outcomes. Fifth, we did not include a heterosexual comparison, thus we cannot make conclusions about whether, for example, transgender individuals experienced more or less family conflict than the general population. Finally, this is a cross-sectional study and longitudinal data are needed to examine the effects of sociopolitical changes over time.

\section{Conclusions}

This study provides insights about safety and related concerns in relation to a specific event in the social and political landscape - the 2016 presidential election. Findings document profound concern about personal safety and wellbeing among transgender individuals, and about the safety and wellbeing of other marginalized groups. At the same time, a number of strengths were reflected across respondent narratives. Many respondents described embracing the support of friends and family, for both comfort and as a source of continued advocacy. Others described a renewed commitment to honoring and celebrating their own identities, despite perceived pressures to become less visible. Factors such as family support, peer support, and identity pride may help buffer the impact of minority stress on psychological health (Bockting et al. 2013), and warrant future investigation. Our findings also point to ways in which clinicians and community groups can bolster existing strengths and resiliencies among transgender individuals to provide buffers in the face of anti-LGBTQ policies and legislation by the Trump administration.

High levels of concerns about safety and discrimination were reported among transgender and gender-nonconforming individuals in light of the social and political climate associated with the 2016 presidential election. Our findings underscore the importance of continuing existing supportive policies, and enacting policies on federal and state levels to document antitrans violence and establish legal rights for transgender people. In communities that are perceived as having high levels of tolerance, rates of depression among transgender individuals are three times lower (Owen-Smith et al. 2016), suggesting sizable health benefits of supportive policies. Although supportive policies are not enough to fully assure the safety and wellbeing of transgender and gender-nonconforming individuals, policies do send a structural message affirming the lives and safety of transgender individuals.

\section{Acknowledgments}

The authors gratefully acknowledge all those who participated in their survey. The authors would also like to thank Lauren Jow for her edits and comments. Research reported in this publication was supported in part by San José State University, the University of Illinois at Chicago, and Columbia University School of Nursing.

\section{Author Disclosure Statement}

No competing financial interests exist.

\section{References}

American Civil Liberties Union. (2017). Legislation affecting LGBT rights across the country. New York, NY. Retrieved May 27, 2017, from https://www.aclu.org/other/legislation-affecting-lgbt-rights-acrosscountry

Bockting WO, Miner MH, Swinburne Romine RE, et al. (2013). Stigma, mental health, and resilience in an online sample of the US transgender population. Am J Public Health. 103, 943-951.

Braun V, Clarke V. (2013). Successful Qualitative Research: A Practical Guide for Beginners. (SAGE Publications, Ltd., London.)

Creswell JW, Poth CN. (2018). Qualitative Inquiry \& Research Design: Choosing Among Five Approaches. (SAGE Publications, Thousand Oaks, CA.)

Frost DM, Fingerhut AW. (2016). Daily exposure to negative campaign messages decreases same-sex couples psychological and relational well-being. Group Processes Intergroup Relations. 19, 477-492.

Gallup. (2017, May 15). US support for gay marriage edges to new high. Retrieved from www.gallup.com/poll/210566/support-gaymarriage-edges-new-high.aspx (accessed May 28, 2017)

Hatzenbuehler ML. (2009). How does sexual minority stigma "get under the skin?" A psychological mediation framework. Psychol Bull. 135, 707-730.

Hatzenbuehler ML, Pachankis JE. (2016). Stigma and minority stress as social determinants of health among lesbian, gay, bisexual, and transgender youth. Pediatr Clin North Am. 63, 985-997.

Hendricks ML, Testa RJ. (2012). A conceptual framework for clinical work with transgender and gender nonconforming clients: An adaptation of the Minority Stress Model. Prof Psychol. 43, 460-467.

Herek GM. (2015). Beyond "homophobia": Thinking more clearly about stigma, prejudice, and sexual orientation. Am J Orthopsychiatry. 85, S29-S37.

Hirsch JK, Kaniuka A, Brooks B, Hirsch KK, Cohn TJ, Williams SL. (2017). Postelection distress and resiliency in LGBTQ communities. Retrieved May 21, 2017, from http://www.apadivisions.org/division-44/ publications/newsletters/division/2017/03/postelection-distress.aspx

Hughes TL, Wilsnack SC, Kristjanson AF. (2015). Substance use and related problems among U.S. women who identify as mostly heterosexual. BMC Public Health. 15, 1-8.

Kenagy GP, Bostwick WB. (2005). Health and social service needs of transgender people in Chicago. Int J Transgender. 8, 57-66.

Levitt HM, Ippolito MR. (2013). Being transgender: Navigating minority stressors and developing authentic self-presentation. Psychol Women Q. 38, 46-64. 
Levy BL, Levy DL. (2016). When love meets hate: The relationship between state policies on gay and lesbian rights and hate crime incidence. Soc Sci Res. 61, 142-159.

Martin-Storey A. (2016). Gender, sexuality, and gender nonconformity: Understanding variation in functioning. Child Dev Perspect. 1-6.

Meyer IH. (1995). Minority stress and mental health in gay men. J Health Soc Behav. 36, 38-56.

Meyer IH. (2003). Prejudice, social stress, and mental health in lesbian, gay, and bisexual populations: Conceptual issues and research evidence. Psychol Bull. 129, 674-697.

Meyer IH. (2015). Resilience in the study of minority stress and health of sexual and gender minorities. Psychol Sex Orient Gender Divers. 2, 209-213.

Miller LR, Grollman EA. (2015). The social costs of gender nonconformity for transgender adults: Implications for discrimination and health. Sociol Forum (Randolph N J). 30, 809-831.

Movement Advancement Project. (2017). Mapping transgender equality in the United States (pp. 1-24). Boulder, CO. Retrieved from http://www.lgbtmap.org/mapping-trans-equality

Owen-Smith AA, Sineath C, Sanchez T, et al. (2016). Perception of community tolerance and prevalence of depression among transgender persons. J Gay Lesbian Ment Health. 21, 64-76.

Perez-Brumer A, Hatzenbuehler ML, Oldenburg CE, Bockting W. (2015). Individual- and structural-level risk factors for suicide attempts among transgender adults. Behav Med. 41, 164-171.

Reisner SL, Hughto JM, Dunham EE, et al. (2015). Legal protections in public accommodations settings: A critical public health issue for transgender and gender-nonconforming people. Milbank Q. 93, 484-515.

Reisner SL, White Hughto JM, Gamarel KE, et al. (2016). Discriminatory experiences associated with posttraumatic stress disorder symptoms among transgender adults. J Couns Psychol. 63, 509-519.

Riggle EDB, Rostosky SS, Horne S. (2010). Does it matter where you live? Nondiscrimination laws and the experiences of LGB residents. Sex Res Soc Policy. 7, 168-175.
Singh A, Hays D, Watson L. (2011). Strength in the face of adversity: Resilience strategies of transgender individuals. J Couns Dev. 89, 20-27.

Singh AA, McKleroy VS. (2011). "Just getting out of bed is a revolutionary act": The resilience of transgender people of color who have survived traumatic life events. Traumatology. 17, 34-44.

Stone AL. (2016). Rethinking the tyranny of the majority: The extralegal consequences of anti-gay ballot measures. Chapman Law Rev. 19, 219-240.

Stotzer RL. (2009). Violence against transgender people: A review of United States data. Aggress Violent Behav. 14, 170-179.

Testa RJ, Habarth J, Peta J, et al. (2015). Development of the gender minority stress and resilience measure. Psychol Sex Orient Gender Divers. 2, 65-77.

Testa RJ, Sciacca LM, Wang F, et al. (2012). Effects of violence on transgender people. Prof Psychol. 43, 452-459.

The GenIUSS Group. (2013). Gender-related measures overview. The Williams Institute (pp. 1-10). Los Angeles, CA. Retrieved from https://williamsinstitute.law.ucla.edu/wp-content/uploads/GenIUSSGender-related-Question-Overview.pdf

Veldhuis CB, Drabble L, Riggle EDB, et al. (2018). "We won't go back into the closet now without one hell of a fight" : Effects of the 2016 presidential election on sexual minority women's and gender minorities' stigma-related concerns. Sex Res Soc Policy. $15,12-24$.
Address correspondence to:

Cindy B. Veldhuis, PhD School of Nursing Columbia University 630 West 168th Street New York, NY 10032

E-mail: c.veldhuis@columbia.edu 